\title{
Docentes y estudiantes de diseño industrial del Centro Universitario UAEM Valle de Chalco, adaptaciones educativas para proyectos terminales de grado ante el COVID-19
}

\begin{abstract}
Omar Eduardo Sánchez Estrada ${ }^{a}$, Josué Deniss Rojas Aragón ${ }^{b}$, karla Georgina Pérez Gonzalez $^{\text {c, }}$ Mario Gerson Urbina Pérez ${ }^{d}$

${ }^{a}$ Universidad Autónoma del Estado de México, Estado de México, México, omarseuaem@yahoo.com.mx, ${ }^{b}$ Universidad Autónoma del Estado de México, Estado de México, México, ' ${ }^{c}$ Universidad Pedro de Gante, Estado de México, México, graham.georginaglez@yahoo.com.mx, ${ }^{\mathrm{d}}$ Universidad Autónoma del Estado de México, Estado de México, gerurb@suv.udg.mx
\end{abstract}

\begin{abstract}
Resumen
La pandemia generada por el COVID-19 ha cambiado las actividades académicas en todo el mundo, la mayoría de los estudiantes y docentes enfrentan un reto importante de comunicación, administración y gestión, así como de generación y aplicación del conocimiento para lograr el cumplimiento de objetivos, estrategias didácticas, prácticas de taller y laboratorio, visitas guiadas e instrumentos de evaluación entre otros. Para los diseñadores industriales que cursan los dos últimos semestres de la licenciatura en Diseño Industrial no es la excepción, ya que deben formalizar con un protocolo de investigación lo que será su proyecto terminal de grado. Por consiguiente, deben proponer proyectos innovadores que impacten favorablemente en un entorno determinado. El presente trabajo tiene como propósito, identificar y analizar estrategias que integran la estructura de enseñanza aprendizaje de los diseñadores industriales para facilitar su adaptación educativa en la propuesta, planeación y desarrollo de proyectos terminales de grado, con la finalidad de lograr mayor impacto en múltiples áreas, específicamente en la médica, rehabilitación, saneamiento, psicología $y$ entretenimiento.
\end{abstract}

Se utilizó la teoría de la información denominada "distancia de Hamming" como herramienta para verificar si el valor estimado real es congruente con el valor estimado ideal, valores numéricos obtenidos de la conceptualización e identificación de factores relevantes para proponer proyectos terminales de diseño en contextos diferenciados. Como resultado se obtuvieron nuevos parámetros para educar más asertivamente hacia un pensamiento innovador 
Docentes y estudiantes de Diseño Industrial del Centro Universitario UAEM Valle de Chalco, adaptaciones educativas para proyectos terminales de grado ante el COVID-19

de los diseñadores industriales, aplicando requerimientos de diseño, con base en las características de interacción, uso, función y adaptabilidad restringidas por el COVID-19.

Palabras clave: Diseño industrial, adaptaciones educativas, COVID-19.

\section{Introducción}

Los educadores en diseño están utilizando nuevas formas de transferir el conocimiento, las redes sociales son usadas para tener contacto inmediato con estudiantes, directivos, administrativos y otros relacionados con la estructura de enseñanza aprendizaje. La educación en general está sufriendo cambios sobre la marcha, adaptándose a las necesidades preestablecidas y aquellas emergentes. La Organización de la Naciones Unidas para la Educación la Ciencia y la Cultura (UNESCO), a través del Instituto Internacional para la Educación Superior en América Latina y el Caribe (IESALC), presentó el 3 de junio del 2020 el informe, la garantía de calidad y los criterios de acreditación en la evaluación superior. Uno de los puntos considerado vector fundamental relacionado con la garantía de calidad, se refiere a los logros académicos de los estudiantes, hasta llegar a la calidad en el respeto a la diversidad. Dicho documento diseñado por el director del IESALC Francesc Pedró y la investigadora de la Universidad de Cambridge Aliandra Barlete, abordan conceptos como la generación de confianza en la cual los métodos de enseñanza cumplen las expectativas o por lo menos se encuentran en los estándares mínimos, además de establecer puntos de referencia, la autoregulación y la motivación de las universidades por obtener mejor reconocimiento mundial.

Bajo estos términos y otros particulares del Centro Universitario UAEM Valle de Chalco, en donde se hizo la presente investigación, encontramos que la licenciatura en diseño industrial se concluye en 10 semestres, de los cuales en noveno específicamente, los estudiantes presentan un anteproyecto (proyecto terminal), que debe atender una problemática local, estatal, nacional o global, asimismo, resolver requerimientos técnicos, estéticos, ergonómicos, de sostenibilidad y contexto para entonces obtener el grado. Sin embargo, la pandemia provocada por el COVID-19 ha transformado la vida personal, social, laboral y educativa de la comunidad universitaria en todas sus actividades, propuestas y proyecciones. 


\section{Adaptaciones educativas}

Se sabe que la respuesta de las universidades ante el COVID-19, es el aislamiento al interior de sus instalaciones y un inmediato desarrollo de planes de estudio en línea. Una prueba de respuesta institucional (Wu, 2020), la cual se estructura en una modalidad en línea y no necesariamente en una pedagogía en línea, además de ser un golpe fuerte a las universidades, estudiantes y docentes con escasos recursos, así como el acceso limitado a la tecnología e internet (Zhong, 2020). Cabe destacar que el uso de las redes sociales y plataformas virtuales para las reuniones a distancia crecen de manera exponencial, alineando de alguna manera la educación hacia esos terrenos (Morris, 2020; Pérez, 2020). Por tanto, cada institución ha respondido de manera diferente, sin comprender los cambios que evolucionan de manera acelerada y que integran la digitalización del curriculum.

En el estudio realizado por Crawford, Butler, Rudolph y Glowatz (2020), presentan una valoración de la agilidad de respuesta de 20 instituciones de educación superior en el mundo ante la pandemia. En la Tabla 1. Se recupera lo más importante de cada país.

Tabla 1. Respuesta de las universidades en el mundo ante la pandemia

\begin{tabular}{|c|c|c|c|c|}
\hline \multicolumn{5}{|c|}{ Características generales que afectaron la respuesta educativa en determinada región } \\
\hline $\begin{array}{l}\text { Región } \\
\text { Europea }\end{array}$ & $\begin{array}{c}\text { Alemania } \\
\text { Todas las } \\
\text { universidades } \\
\text { en línea } \\
\text { No hay ley } \\
\text { para presentar } \\
\text { titulaciones } \\
\text { en línea }\end{array}$ & $\begin{array}{l}\text { Italia } \\
\text { Todas las } \\
\text { universidades en } \\
\text { línea } \\
\text { Exámenes } \\
\text { terminales en } \\
\text { línea }\end{array}$ & $\begin{array}{c}\text { Irlanda } \\
\text { Todas las } \\
\text { universidades } \\
\text { en línea } \\
\text { Hasta el fin de } \\
\text { año académico }\end{array}$ & $\begin{array}{c}\text { Reino Unido } \\
\text { Todas las universidades en línea } \\
\text { Apoyo a estudiantes } \\
\text { internacionales } \\
\text { Incertidumbre por (Brexit) }\end{array}$ \\
\hline $\begin{array}{l}\text { Región de } \\
\text { las } \\
\text { Américas }\end{array}$ & $\begin{array}{l}\text { EE.UU. } \\
\text { Algunas } \\
\text { universidades } \\
\text { (enseñanza en } \\
\quad \text { línea) }\end{array}$ & $\begin{array}{c}\text { Brasil } \\
\text { Algunas } \\
\text { universidades } \\
\text { (enseñanza en } \\
\text { línea). }\end{array}$ & \multicolumn{2}{|c|}{$\begin{array}{l}\underline{\text { Chile }} \\
\text { Algunas universidades (enseñanza en línea) } \\
\text { Cárcel por faltas a la cuarentena }\end{array}$} \\
\hline $\begin{array}{l}\text { Región } \\
\text { Africana }\end{array}$ & $\begin{array}{c}\text { Nigeria } \\
\text { Algunas } \\
\text { universidades } \\
\text { (enseñanza en } \\
\text { línea) }\end{array}$ & \multicolumn{3}{|c|}{$\begin{array}{l}\text { Sudáfrica } \\
\text { rsidades (enseñanza en línea) } \\
\text { n en casa, en alguna universidad la } \\
\text { ón permaneció habilitada }\end{array}$} \\
\hline $\begin{array}{l}\frac{\text { Región del }}{\text { Mediterrán }} \\
\text { eo Oriental } \\
\end{array}$ & $\begin{array}{l}\text { Egipto } \\
\text { Todas las } \\
\text { universidades } \\
\text { en línea }\end{array}$ & $\begin{array}{l}\text { Jordania } \\
\text { Algunas } \\
\text { universidades } \\
\text { (enseñanza en } \\
\quad \text { línea) } \\
\text { Acceso a Internet } \\
\text { de alta velocidad }\end{array}$ & \multicolumn{2}{|c|}{$\begin{array}{c}\text { Emiratos Árabes Unidos } \\
\text { Todas las universidades enseñanza en línea } \\
\text { Smart University fue la primera universidad } \\
\text { electrónica en los EAU } \\
\text { No hay estrategia en línea sino emergencia en línea } \\
\text { Posición única para continuar } \\
\text { con la enseñanza en línea }\end{array}$} \\
\hline
\end{tabular}


Docentes y estudiantes de Diseño Industrial del Centro Universitario UAEM Valle de Chalco, adaptaciones educativas para proyectos terminales de grado ante el COVID-19

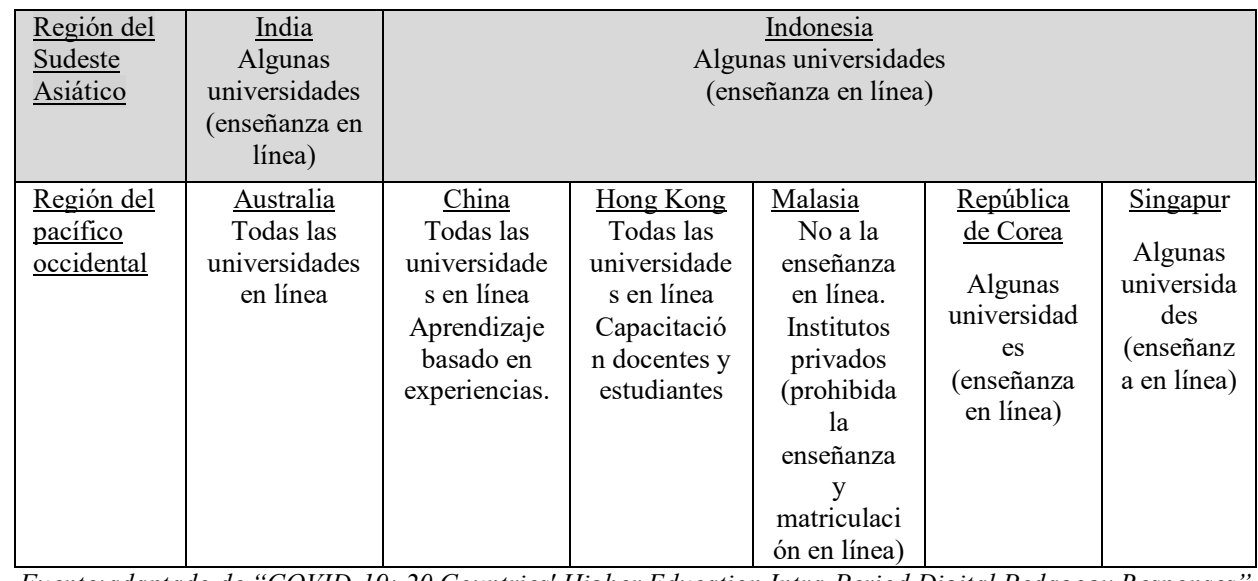

Fuente:adaptado de "COVID-19: 20 Countries' Higher Education Intra-Period Digital Pedagogy Responses"

(Crawford et al. 2020).

Cabe destacar que la mayoría de las universidades han cerrado y las economías nombradas desarrolladas están reportando una gran cantidad de infectados por millón, ninguna de ellas considera extender la suspensión para todo el semestre, se sigue trabajando en línea con excepción de los Estados Unidos de América y los proveedores de educación en línea, tienen una magnífica oportunidad para seguir creciendo. Por tanto, la consolidación de una educación a distancia no será inmediata, ya que se carece de infraestructura, equipamiento para el trabajo en casa y la accesibilidad a internet, además que en algunos países el acceso a la red está intervenido por su gobierno (Crawford et al. 2020).

Por otro lado, se han desarrollado respuestas más elaboradas por ejemplo, el gobierno Chino creó una estrategia de educación en línea a gran escala. Uno de los puntos a considerar para la educación en general es, sumarse a las cinco enseñanzas básicas: a) inteligencia; b) trabajo; c) estética; d) moral; y e) deportiva (Zhou et al. 2020). Además de lo siguiente:

1. Coordinar el currículum y la educación relacionada con la epidemia. Considerando el patriotismo, prevención, educación para la vida, seguridad pública y mental.

2. Guiar a los estudiantes a comprender correctamente la relación entre las personas, sociedad y naturaleza.

3. Coordinar el trabajo de los profesores principales y los que apoyan el eje del plan de estudios, organizar a los docentes destacados en el uso de la tecnologías de la información y comunicación TIC's para la grabación de cursos, provisión de recursos y orientación docente en línea, asimismo organizar a los docentes para participar en orientación de estudiantes en el aprendizaje en línea. 
4. Se prohíbe exigir a los docentes que transmitan o grabar clases en vivo, no debe ser obligatorio para los estudiantes (Ministerio de Educación de la República Popular de China, 2020).

También diferentes universidades y empresas han desarrollado proyectos para contrarrestar el impacto negativo de la pandemia. Por ejemplo, en nuestra casa de estudios la Universidad Autónoma del Estado de México (UAEMex). Se desarrollan cápsulas para tomar muestras y cajas de intubación para reducir el riesgo de contagio por COVID-19.

La empresa Koniku afirma que la pieza tecnológica más avanzada en el mundo es el software. Logra fusionar neuronas biológicas de tecnología de silicio completa con detección de olores, clasificación y aprendizaje biológico real. Con un sistema de vigilancia de olores sin contacto, ha encontrado la aplicabilidad de seguridad sanitaria en los aeropuertos.

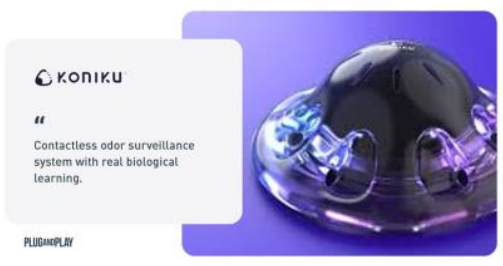

Fig. 1 Koniku Pieza tecnológica, detección de olores clasificación de aprendizaje biológico sin contacto.

Fuente. PLUGANDPLAY (2020).

Positiv Air tecnología patentada- Ao-Air trabaja con ventiladores para crear presión positiva y generar aire limpio, permite que el usuario respire libremente sin necesidad de sellar la boca y la nariz, el sistema logra que el aire limpio y frío escape cómodamente de la máscara alrededor de la cara cuando el flujo de salida continuo y unidireccional mantiene el aire exterior afuera.

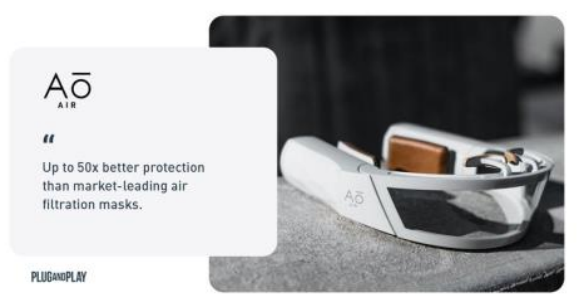

Fig.2 Ao-air tecnología para generar aire limpio. Fuente: PLUGANDPLAY (2020).

VENTEC LIFE desarrolló un sistema respiratorio integrado VOCSN, ventiladores portátiles para redefinir la atención de problemas respiratorios, integra cinco dispositivos 
Docentes y estudiantes de Diseño Industrial del Centro Universitario UAEM Valle de Chalco, adaptaciones educativas para proyectos terminales de grado ante el COVID-19

médicos diferentes (ventilador, concentrador de oxígeno, apoyo para la tos, succión y nebulizador)

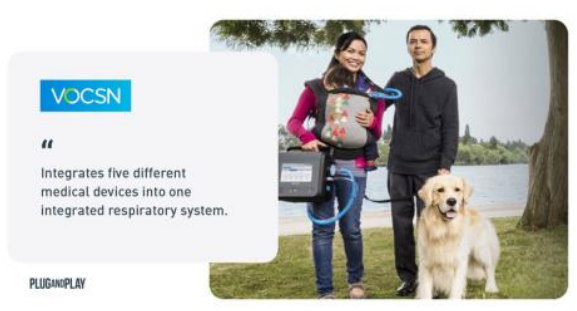

Figura 3. VOCSN sistema respiratorio integrado.

Fuente: PLUGANDPLAY (2020).

\section{Conceptuación de los proyectos terminales}

Como resultado del trabajo realizado durante 10 semestres, los estudiantes deben generar y aplicar conocimiento a partir del anteproyecto expuesto en octavo semestre, el cual se define a través de las diferentes alternativas de desarrollo, entre las cuales se destacan: equipos especializados, mobiliario, ergonomía, herramientas y accesorios, teoría del diseño, equipo médico, joyería y entretenimiento. La última generación periodo febrero agosto de 2020-A eligió trabajar con la siguientes opciones:

Porcentajes de selección de las áreas para desarrollar trabajo terminal de grado
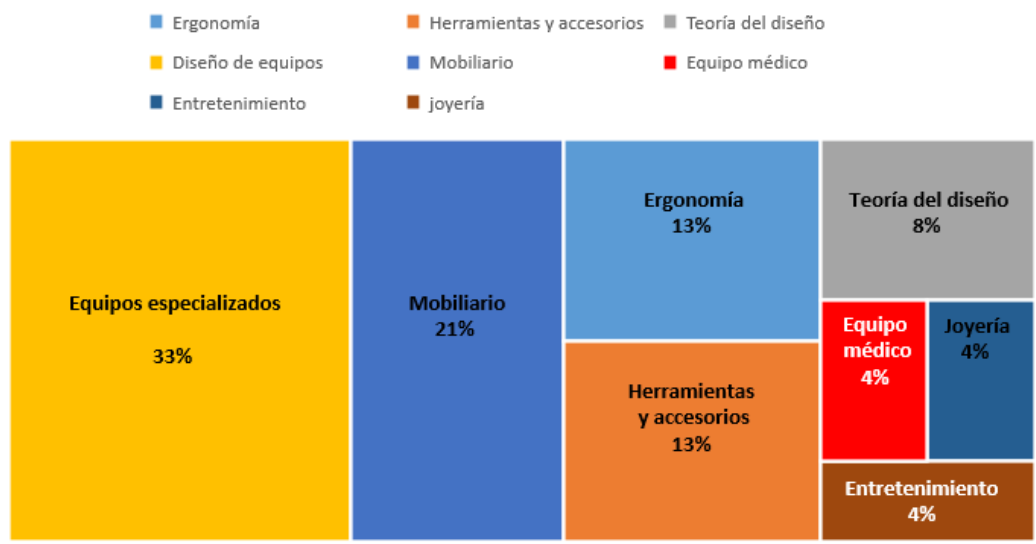

Fig. 4 Selección del área para el desarrollo del trabajo terminal de grado de los estudiantes de la licenciatura en Diseño Industrial del Centro Universitario UAEM Valle de Chalco.

Fuente: Propia 
Es evidente que la elección de las temáticas referentes a equipos especializados y mobiliario, son los ejes de proyección más recurrentes y el equipo médico o los proyectos enfocados en resolver necesidades globales, no entran en la preferencia de los alumnos que están por graduarse. Las Unidades de aprendizaje: proyecto Integral de diseño industrial I y II correspondientes a los semestres 9 y 10, se caracterizan por guiar al estudiante hacia los proyectos que cumplan con los criterios delimitados por la teoría, práctica, filosofía, sostenibilidad o aplicación del diseño industrial, partiendo desde la implantación de la propuesta, desarrollo de la metodología de la investigación y la de diseño, análisis y definición del usuario, procesos sostenibles, selección de materiales, diseño y validación de instrumentos si es el caso y la representación de los resultados en sus diferentes modalidades. Es por ello, que se definieron los conceptos que orientarán a los alumnos a elegir proyectos terminales y las posibles combinaciones de variables que pueden trabajar sin descuidar la parte técnica, estética, funcional y simbólica de las necesidades actuales. (la numeración de los conceptos se asignó únicamente para su identificación).

Tabla 2. Guía de conceptos para la elección de proyectos terminales de diseño industrial ante el COVID-19

\begin{tabular}{|c|c|c|c|}
\hline Área médica & $\underline{\text { Recursos }}$ & $\begin{array}{l}\text { Proyectos } \\
\text { epecializados }\end{array}$ & Teoría del diseño \\
\hline $\begin{array}{l}\text { 1. Protección } \\
\text { personal }\end{array}$ & $\begin{array}{l}\text { 2. Tratamiento y ahorro } \\
\text { de agua }\end{array}$ & $\begin{array}{l}\text { 3. Aplicación de } \\
\text { materiales }\end{array}$ & 4. Enfoques de diseño \\
\hline $\begin{array}{l}\text { 5. Equipo de } \\
\text { atención y } \\
\text { prevención }\end{array}$ & $\begin{array}{l}\text { 6. Usos y aplicaciones de } \\
\text { la energía } \\
\text { (solar, eólica, térmica, } \\
\text { eléctrica, } \\
\text { electromagnética, física y } \\
\text { mecánica) }\end{array}$ & $\begin{array}{l}\text { 7. Sistemas de } \\
\text { detección }\end{array}$ & 8. Sostenibilidad \\
\hline $\begin{array}{l}\text { 9. Sistemas de } \\
\text { aislamiento }\end{array}$ & $\begin{array}{c}\text { 10. Tratamiento del } \\
\text { ambiente }\end{array}$ & $\begin{array}{l}\text { 11. Ergonomía } \\
\text { aplicada }\end{array}$ & 12. Percepción visual \\
\hline $\begin{array}{l}\text { 13. Ergonomía } \\
\text { para hospitales }\end{array}$ & 14. Ergonomía ambiental & 15. Transporte & 16. Teoría del color \\
\hline $\begin{array}{l}\text { 17. Mobiliario } \\
\text { para hospitales }\end{array}$ & $\begin{array}{c}\text { 18. Sistemas de } \\
\text { tratamiento de desechos }\end{array}$ & $\begin{array}{l}\text { 19. Estaciones de } \\
\text { trabajo }\end{array}$ & 20. Ergonomía cognitiva \\
\hline $\begin{array}{l}\text { 21. Máquinas y } \\
\text { herramientas }\end{array}$ & 22. Reciclaje & $\begin{array}{l}\text { 23. Sistemas de } \\
\text { entretenimiento }\end{array}$ & 24. Proxémica \\
\hline $\begin{array}{l}\text { 25. Equipo } \\
\text { especializado }\end{array}$ & 26. Reuso y reutilizacón & $\begin{array}{c}\text { 27. Envase, } \\
\text { empaque y } \\
\text { embalaje }\end{array}$ & $\begin{array}{l}\text { 28. Prospectiva del } \\
\text { diseño }\end{array}$ \\
\hline
\end{tabular}

Fuente: Propia

Para validar la investigación se utilizó una rama de la teoría matemática y de las ciencias de la computación. Claude E. Shannon (ingeniero electrónico y matemático) y Warren Weaver (biólogo e informático) publicaron en 1948 la Teoría Matemática de la Comunicación (The Mathematical Theory of Communication). La teoría demostró que todas las fuentes de información (telégrafo eléctrico, teléfono o la gente al hablar, etcétera) pueden medirse y 
que los canales de comunicación tienen una unidad de medida similar, determinando la velocidad máxima de transferencia o capacidad de canal.

Dicha teoría se denomina distancia de Hamming la cual describe lo siguiente: efectividad de los códigos de Bloque (técnicas utilizadas para transformar un conjunto de datos binarios "N" en otro un tanto más largo "K") depende de la diferencia entre una palabra de código válida y otra. Cuanto mayor sea esta diferencia, menor es la posibilidad de que un código válido se transforme en otro código válido por una serie de errores. A esta diferencia se le denomina "distancia de Hamming" y se define como el número de bits que tienen que cambiarse para transformar una palabra de código válida en otra palabra de código válida. Si dos palabras de código difieren en una distancia d, se necesitan d errores para convertir una en la otra (Hamming, 1950, 90). De esta manera, la distancia de Hamming se inserta en este estudio como una forma de validar la información obtenida en las encuestas aplicadas a docentes especialistas del área, permitiendo verificar si la distancia entre el valor estimado real es congruente con el valor estimado ideal.

Por consiguiente, se utilizó la distancia de Hamming como herramienta para validar las diferentes posturas emitidas por los docentes. Se analizaron los niveles de frecuencia y porcentajes, así como la ponderación en una escala de 0 a 10 para los conceptos de elección de proyectos terminales, de esta manera, se obtuvo el vector real del nivel de frecuencia. Posteriormente, se aplicó el siguiente algoritmo para definir el vector ideal: la distancia de Hamming se define de la siguiente manera:

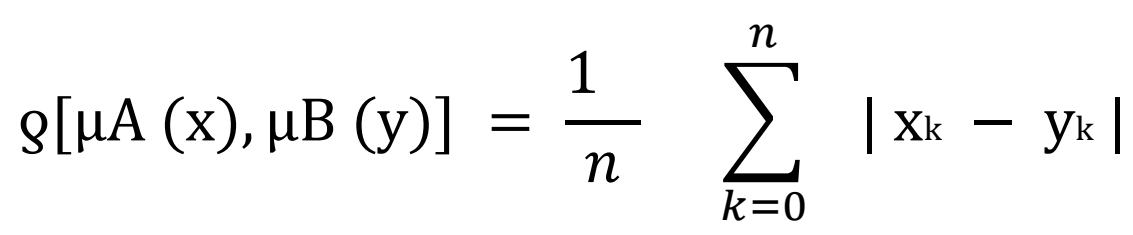

donde:

$\mathrm{A}(\mathrm{x})$ es el vector de los promedios reales en cada nivel de los criterios sugeridos para elegir temática proyectual.

$\mathrm{B}(\mathrm{y})$ es el vector de los promedios estimados en cada nivel de los criterios sugeridos para elegir temática proyectual.

$\mu_{\mathrm{A}(\mathrm{x})}$ define a los atributos del conjunto $\mathrm{A}(\mathrm{x})$.

$\mu_{\mathrm{B}(\mathrm{y})}$ define a los atributos del conjunto $\mathrm{B}(\mathrm{y})$.

$\mathrm{X}_{\mathrm{k}}$ es el k-ésimo atributo del conjunto $\mathrm{A}(\mathrm{x})$.

$\mathrm{Y}_{\mathrm{k}}$ es el k-ésimo atributo del conjunto $\mathrm{B}(\mathrm{y})$.

$\mathrm{n}$ es el total de atributos. 


\section{Resultados}

A continuación se presenta la Gráfica 1. con el análisis de frecuencia de los vectores

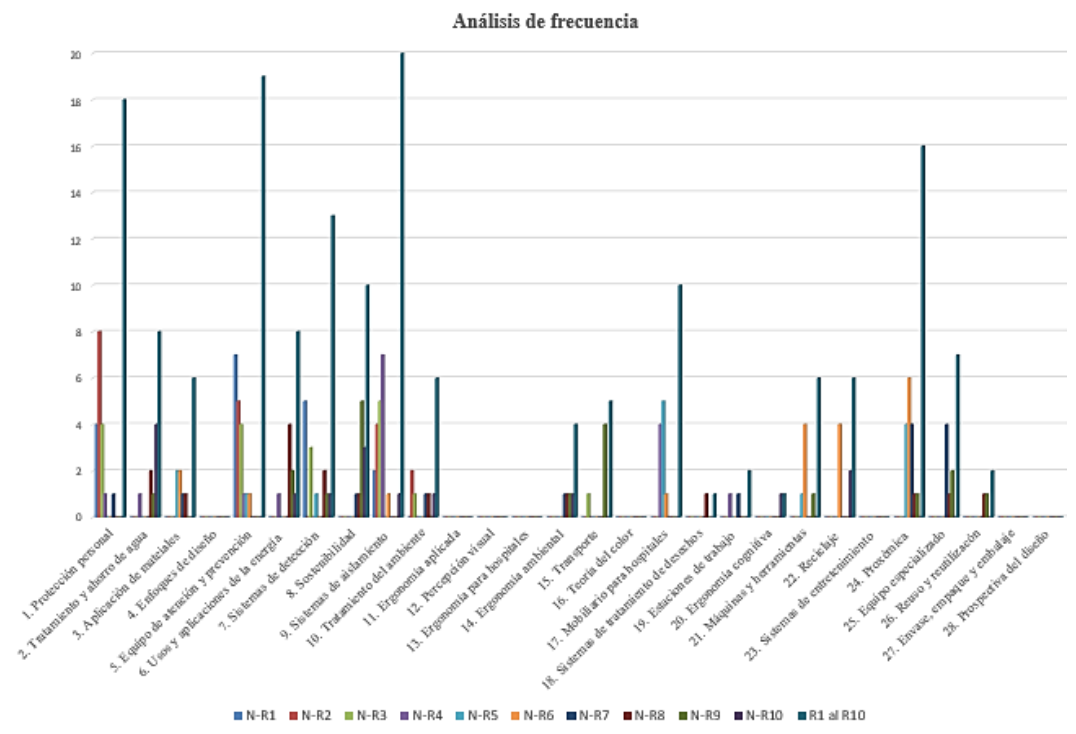

Gráfica 1. Análisis de frecuencia

Fuente:propia

El concepto elegido con mayor frecuencia fue (N1), Por lo tanto, se puede afirmar que los conceptos elegidos más relevantes tienen un grado de fiabilidad bastante aceptable. Cabe señalar que un valor numérico en la escala de 0 a 10 , lo más cercano al valor 0 se considera un resultado altamente satisfactorio cuando se habla de la distancia de Hamming. Por lo que, para hablar de un proceso de validación de los resultados, este valor no deberá sobrepasar él (3). La gráfica 2 describe el promedio distancia obtenido de (1.9) el cual se obtuvo de la aplicación de la fórmula considerando todos los datos en cada concepto. 
Docentes y estudiantes de Diseño Industrial del Centro Universitario UAEM Valle de Chalco, adaptaciones educativas para proyectos terminales de grado ante el COVID-19

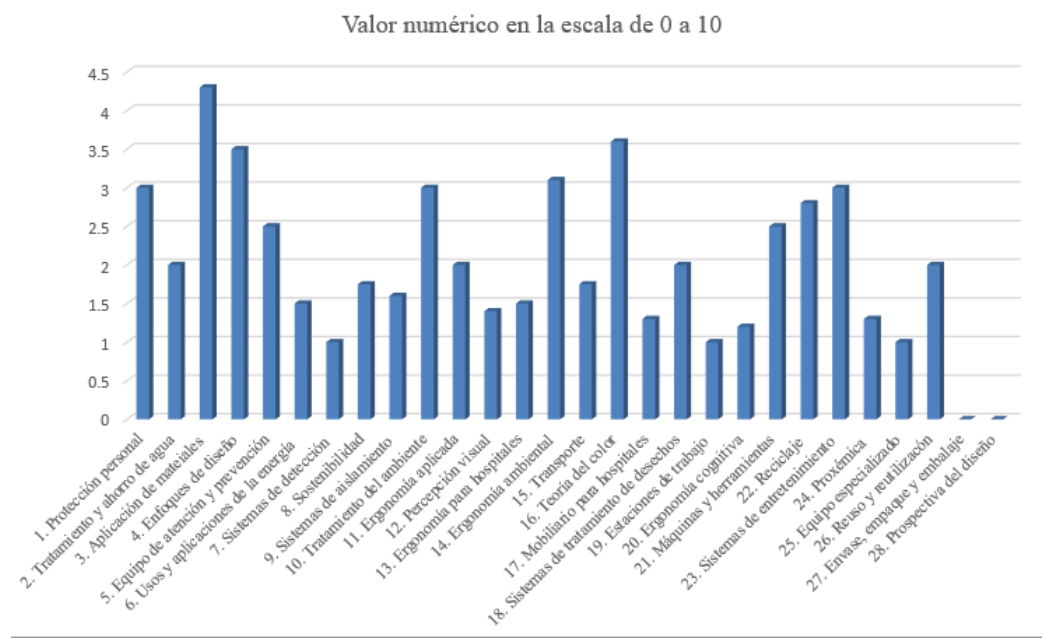

Gráfica 2. Valor numérico en la escala 0 a 10, sumatoria 55.6/28=(1.9)promedio distancia Fuente:propia

Por tanto, el nivel de importancia de los conceptos definidos es la siguiente:

(1)---1. Protección personal

(2)---25. Equipo especializado

(3)---7. Sistemas de detección

(4)---17. Mobiliario para hospitales

(5)---5. Equipo de atención y prevención

(6)---6. Usos y aplicaciones de la energía

(7)---25. Equipo especializado

(8)---21. Máquinas y herramientas

(9)---15. Transporte

(10)--14. Ergonomía ambiental

\section{Conclusiones}

Los docentes y estudiantes deberán tomar mejores decisiones para trabajar con un contexto completamente diferente al que se vivía antes de la pandemia, los proyectos terminales son el punto de partida para hacer un ejercicio de profesionalización ante situaciones de emergencia y con requerimientos de diseño complejos e inmediatos. 


\section{Referencias}

Crawford, J., Butler-Henderson, K., Rudolph, J., \& Glowatz, M. (2020). COVID-19: 20 Countries' Higher Education Intra-Period Digital Pedagogy Responses. Journal of Applied Teaching and Learning (JALT), 3(1).

Hamming, R. W. (1950). Error detecting and error correcting codes. The Bell System technical journal, 29 (2):147-160.

Morris, I. (2020). COVID-19 makes data connectivity as critical as toilet paper. Light Reading. Recuperado de: https://www.lightreading.com/optical-ip/fttx/COVID-19-makes-dataconnectivity-ascritical-as-toilet-paper/d/d-id/758195

Perez, S. (2020). Neilsen explains how COVID-19 could impact media usage across the US. TechCrunch. Recuperado de: https://techcrunch. com/2020/03/17/nielsen-explains-how-COVID19-couldimpact-media-usage-across-the-u-s/

PLUGANDPLAY. (2020) COVID-19 y la carrera en tecnología. Recuperado de: https://www.plugandplaytechcenter.com/resources/covid-19-and-race-startup-technology

UNESCO (2020). IESALC lanza estudio sobre calidad y acreditación en la educación superior. Venezuela:IESALC, UNESCO, Educación para todas las personas. Recuperado de: http://www.iesalc.unesco.org/2020/06/03/iesalc-lanza-estudio-sobre-calidad-y-acreditacion-en-laeducacion-superior/

Wu, Z. (2020). How a top Chinese university is responding to coronavirus. In World Economic Forum.

Zhong, R. (2020). The coronavirus exposes education's digital divide. The New York Times, 17.

Zhou, L., Wu, S., Zhou, M., \& Li, F. (2020). 'School's Out, But Class' On', The Largest Online Education in the World Today: Taking China's Practical Exploration During The COVID-19 Epidemic Prevention and Control As an Example. But Class' On', The Largest Online Education in the World Today: Taking China's Practical Exploration During The COVID-19 Epidemic Prevention and Control As an Example (March 15, 2020). 